\title{
Comparative and functional genomics of the $A B C$ transporter superfamily across arthropods
}

Shane Denecke ${ }^{1 *}$, Ivan Rankić ${ }^{2}$, Olympia Driva ${ }^{1}$, Megha Kalsi ${ }^{1}$, Ngoc Bao Hang Luong ${ }^{1}$, Benjamin Buer ${ }^{3}$, Ralf Nauen ${ }^{3}$, Sven Geibel ${ }^{3}$ and John Vontas ${ }^{1,4}$

\begin{abstract}
Background: The ATP-binding cassette $(A B C)$ transporter superfamily is comprised predominantly of proteins which directly utilize energy from ATP to move molecules across the plasma membrane. Although they have been the subject of frequent investigation across many taxa, arthropod ABCs have been less well studied. While the manual annotation of $A B C$ transporters has been performed in many arthropods, there has so far been no systematic comparison of the superfamily within this order using the increasing number of sequenced genomes. Furthermore, functional work on these genes is limited.

Results: Here, we developed a standardized pipeline to annotate ABCs from predicted proteomes and used it to perform comparative genomics on ABC families across arthropod lineages. Using Kruskal-Wallis tests and the Computational Analysis of gene Family Evolution (CAFE), we were able to observe significant expansions of the ABC-B full transporters (P-glycoproteins) in Lepidoptera and the ABC-H transporters in Hemiptera. RNA-sequencing of epithelia tissues in the Lepidoptera Helicoverpa armigera showed that the 7 P-glycoprotein paralogues differ substantially in their tissue distribution, suggesting a spatial division of labor. It also seems that functional redundancy is a feature of these transporters as RNAi knockdown showed that most transporters are dispensable with the exception of the highly conserved gene Snu, which is probably due to its role in cuticular formation.

Conclusions: We have performed an annotation of the ABC superfamily across $>150$ arthropod species for which good quality protein annotations exist. Our findings highlight specific expansions of ABC transporter families which suggest evolutionary adaptation. Future work will be able to use this analysis as a resource to provide a better understanding of the $A B C$ superfamily in arthropods.
\end{abstract}

Keywords: ABC transporters, Comparative genomics, Gene family evolution, RNAi, Arthropod

\section{Introduction}

The ATP-binding cassette (ABC) superfamily is one of the best studied gene groups in biology [1]. The majority of proteins within the superfamily act as transporters, shuttling a wide variety of endogenous compounds and

\footnotetext{
*Correspondence: shane_denecke@imbb.forth.gr; sdenecke@vet.upenn.edu ${ }^{1}$ Institute of Molecular Biology \& Biotechnology, Foundation for Research \& Technology Hellas, 100 N. Plastira Street, 700 13, Heraklion Crete, Greece Full list of author information is available at the end of the article
}

xenobiotics across lipid membranes. Others, despite their nomenclature, lack transmembrane domains and play other essential cellular functions [2]. Each functioning transporter is made up of two highly conserved nucleotide binding domains (NBDs) and two less conserved transmembrane domains (TMs), which may be found in a single polypeptide (full transporter) or split into multiple subunits that must unite (half transporter) to form functional proteins. They are present in a 
diverse set of taxonomic lineages from "microorganisms to man" [3] and play a variety of physiological functions. Individual $\mathrm{ABCs}$ can be grouped into nine families (named $\mathrm{ABC}-\mathrm{A}$ through $\mathrm{ABC}-\mathrm{I}$ ) based on the sequence homology in their conserved NBD. Although there exists substantial variation within these subgroups, family members share some common allocrites and functions across a range of organisms [4]. Classification of genes into a particular $\mathrm{ABC}$ family can therefore be used as a starting point to investigate potentially interesting genes in non-model organisms.

Less is known about the ABC superfamily in arthropods compared to other taxonomic groups such as mammals or bacteria [4]. However, there has been an increasing effort to understand the molecular biology of arthropods for several reasons. The arthropod phylum is the most diverse of the multicellular eukaryotes and is frequently studied in order to gain fundamental evolutionary insights [5]. Additionally, many arthropod species are pests, having substantial impact in both agriculture and public health. Pesticides including small molecules and protein based biopesticides are the most common way to control these pests, and $A B C$ transporters are thought to play a key role in pesticide biology $[6,7]$. Xenobiotic transporting roles have been suggested for the $A B C-A, A B C-B, A B C-C$, and $A B C-G$ families families $[6,8]$, while members of the $A B C-C$ and ABC-A family often serve as targets for crystal toxins derived from Bacillus thuringiensis [9].

One of the best studied $A B C$ families is the $A B C-B$ clade which consists of both full and half transporters that act on an array of substrates including neutral and cationic amphiphilic compounds [10]. ABC-B full transporters are more commonly known as multidrug resistance proteins (MDRs) or permeability glycoproteins (Pglycoproteins; P-gps) because of their ability to excrete xenobiotic compounds. Human P-gp activity was inhibited by pesticides such as (cypermehtrin, fenvalerate, endosulfan and methyl parathion), suggesting that these compounds may be substrates for this protein [11]. P-gp orthologues in insects have been shown to underpin pesticide resistance in cases such as Heliothis virescens [12] and Drosophila melanogaster [13]. In other cases, genetic manipulation of P-glycoproteins increased or decreased the toxicity of a pesticide in susceptible backgrounds $[14,15]$. However, the genomic trends of P-gp have not been thoroughly investigated.

The ABC-H transporter family is also particularly interesting due to its distribution across species. This family is present in arthropods and a limited number of other species scattered across the tree of life [6]. The majority of arthropods contain fewer than $6 \mathrm{ABC}-\mathrm{H}$ members, but larger numbers were found in two noninsect arthropods Tetranychus urticae and Daphnia pulex along with two hemipteran species (Bemisia tabaci and Diaphorina citri) [16-19]. Structurally, ABC-H proteins are half-transporters and show the same inverted domain architecture as the ABC-G family. The functional characterization of these proteins has been pioneered in Drosophila where the ABC-H transporter Snustorr (Snu) was localized to the integument and shown to transport cuticular hydrocarbons [20]. RNAi knockdown of Snu orthologues in other arthropods has led to lethality due to desiccation, suggesting a similar function [21, 22], but little information exists on other members of the ABC-H family.

Insights into the evolution of a gene family can highlight expansions or contractions which may suggest functional adaptation. Previous studies have taken such an approach by comparing $\mathrm{ABC}$ families across arthropod species, albeit on a limited number (7) of species [6]. In the intervening years the number of sequenced insect genomes has greatly increased thanks in large part to the i5k project and a variety of independent labs sequencing various arthropods from many taxonomic groups [23]. This has led to an avalanche of publications which manually annotate the $A B C$ family of a species using a variety of different methodologies [17, 24, 25]. So far, there has been little effort to systematically compare the $A B C$ transporter superfamily in arthropods. Such a comparative genomic analysis of the $A B C$ superfamily was previously accomplished in plants, [26] and a more recent study has considered the evolution of the Solute carrier (SLC) transporter superfamily in arthropods [27]. To the authors knowledge, only one study has taken this approach in arthropod $\mathrm{ABC}$ transporters, but this work did not distinguish between $\mathrm{ABC}$ families and rather considered only the total number of $\mathrm{ABCs}$ in a species [28].

Here, we extend the knowledge of the $A B C$ transporter superfamily in arthropods through comparative and functional genomics. First, we designed and implemented the $A B C \_s c a n$ algorithm to identify and classify $\mathrm{ABC}$ transporters from the predicted proteome of a species. Comparing the family sizes of different $A B C$ transporter families showed substantial expansions in the lepidopteran $\mathrm{ABC}-\mathrm{B}$ full transporters (ABC-BF; Pglycoproteins) and the hemipteran $\mathrm{ABC}-\mathrm{H}$ transporters. Transcriptomics of relevant epithelial tissues from a model Lepidoptera Helicoverpa armigera and an RNAi screen in a model Hemiptera Nezara viridula were then used to further probe the properties and functions of these expansions.

\section{Materials and methods}

\section{In silico identification of transporters}

Putative $A B C$ transporters were identified in non-model arthropod species using an in silico pipeline dubbed 
$A B C \_s c a n$ that made use of previously annotated ABC transporters to search the predicted protein sets of target species (Fig. 1; https://github.com/shanedenecke/ABC_ scan.git). Unigene protein sets containing a single amino acid sequence per gene were obtained primarily from OrthoDB supplemented with other sources (Table S1). As overestimates or underestimates of the total number of proteins in a species would bias our overall results, we used the Benchmarking Universal Single-Copy Orthologs (BUSCO) method to remove lower quality protein sets [29]. Unigene protein sets for each species were analyzed with BUSCO using the arthropod odb10 lineage (-1 arthropod_odb10) under the proteins setting, and species with a single copy completeness score of below $80 \%$ were excluded from the analysis.

The Hidden Markov Model (HMM) profile corresponding to the $\mathrm{ABC}$ nucleotide binding domain (PF00005) was retrieved from PFAM (http://pfam.xfam. org/fa3zmily/PF00005.23) and used to search predicted protein sets of different arthropod species with the HMMER package v3.2.1 [30]. Candidate transporters with an e-value of less than 10 were kept as potential candidates. These sequences were then used as BLASTP [31] queries (e-value cutoff of 1e-5) against a database comprising proteomes of 4 species (Homo sapiens, Tetranychus urticae, Drosophila melanogaster and Tribolium castaneum) with well annotated ABC sets including family (A-H) annotation [19, 32, 33].

Classification of each candidate sequence into an $\mathrm{ABC}$ family was performed based on their most significant BLAST hits. If 4 out of the top 5 hits were all the same family or the top 3 blast hits were all the same family the candidate was considered part of that family. Alternatively, a candidate was sorted into the family of its top hit if this protein was an $\mathrm{ABC}$ transporter and possessed an e-value greater than 5 orders of magnitude lower than the next best hit. Candidates not meeting these criteria were not considered $\mathrm{ABCs}$ and excluded from this study. Proteins showing ambiguity between the ABC-B full and half transporter subfamilies were categorized based on their number of nucleotide binding domains; $\mathrm{ABC}-\mathrm{BH}$ proteins had one NBD whereas ABC-BF transporters had two or more. The ABC-I family was recently proposed in insects [25], but it was excluded in this study because it was not annotated in any of the model insect proteomes on which the analysis depends.

\section{Analysis of family size over evolutionary time}

The size of each ABC family $(\mathrm{A}-\mathrm{H})$ in each species included in the final analysis were compared in order to identify expansions and contractions over evolutionary time. This was accomplished by first grouping species by their taxonomic group and using a Kruskal-Wallis statistical test with a Bonferroni correction to identify groups with significantly larger or smaller $A B C$ families. This strategy was implemented using the "kruskal" function from the "agricolae" package in $\mathrm{R}$, which calculated $\mathrm{p}$ values reflecting significant differences among taxa and categorized taxa into statistical groups based on their average family sizes.

Family sizes within taxonomic groups were further explored with the Computational Analysis of gene Family Evolution v5 (CAFE; [34]; https://github.com/hahnlab/ CAFE5), which uses phylogenomics and family sizes to

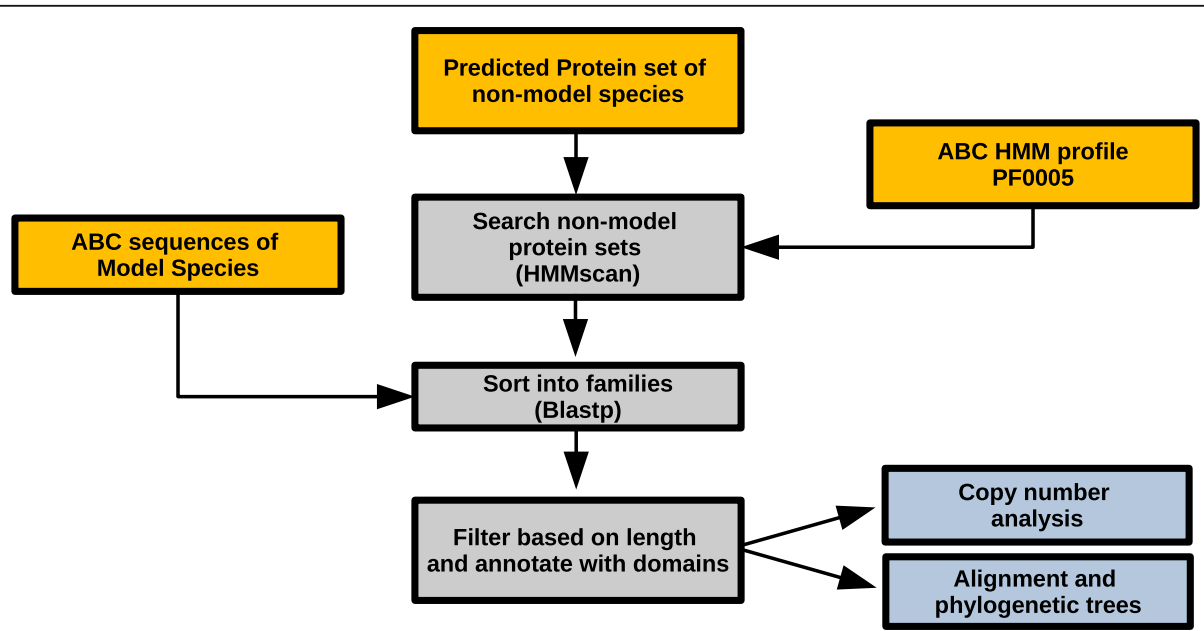

Fig. 1 ABC_scan pipeline schematic. The $A B C \_$scan pipeline can be separated into inputs (orange), actions (grey), and outputs (blue). Predicted protein sets for a given species are fed into the pipeline. In the first step the protein sets are searched with the ABC transporter (PF00005) HMM profile. Next, candidate transporters are sorted into families by blasting against a database of known ABC transporter proteins from $H$. sapiens, $T$. castaneum, D. melanogaster, and T. urticae. Lastly, results are filtered based on their amino acid length and annotated with corresponding metadata before outputting the predicted $A B C$ set in tabular and fasta format 
estimate the timing of gene family evolution. Family sizes for each $A B C$ family in each species were estimated using the $\mathrm{ABC}_{-}$scan pipeline described above. Ultrametric phylogenies were generated for selected subclades of arthropods (Table S2) by first gathering 1:1 orthologues using Orthofinder v2.3.11 [35]. Amino acid sequences from each ortholog group were then aligned with MAFFT [36] and trimmed with Trimal v1.4 [37] using default parameters for the former and the "automated1" algorithm for the latter. These trimmed alignments were then concatenated to form a single sequence for each species and used as an input to RAxML-NG v0.9.0 [38] using the "LG + G8 + F" model for a maximum of 200 bootstraps. Trees were then calibrated using the "chronos" function of the "ape" $\mathrm{R}$ package v5.4-1 using a discrete model and known evolutionary divergences from a previously published study [39]. These trees were then rooted using the "root" function from the same package with designated outgroups (Table S2). All trees were visualized in $\mathrm{R}$ with the ggtree package v2.2.4 [40].

The same procedure (MAFFT, Trimal, RAxML-NG) was used to make protein level phylogenetic trees of selected transporters. For the ABC-B full transporters, sequences from Bombyx mori, Spodoptera frugiperda, Nezara viridula, Danaus plexipus, and Papilio polytes were used. In the ABC-H family N. viridula, Bemisia tabaci, Daphnia pulex, T. urticae, Nilaparvata lugens, Myzus persicae, Rhodnius prolix, and Daktulosphaira vitifoliae were used. Branches with below $50 \%$ bootstrap values were collapsed as polytomic nodes.

\section{Helicoverpa dissections and RNA-seq}

A population of $H$. armigera was obtained from Bayer Crop Sciences and reared in the laboratory for several generations at the Institute of Molecular Biology and Biotechnology (Greece). Larvae were maintained on artificial diet, and adults were fed $10 \%$ sugar solution. All individuals were kept at $16: 8$ light:dark at $26{ }^{\circ} \mathrm{C}$. Dissections of midgut, Malpighian tubules, and central nervous system tissues were performed on L5 larvae using forceps under RNAse free phosphate-buffered saline (PBS) on ice, and RNA was extracted with the GeneJet RNA purification kit (Thermo Scientific). RNA samples were shipped on dry ice to the Macrogen Sequencing facility (Seoul, Korea) and libraries were prepared with the Illumina TruSeq stranded mRNA kit. Libraries were sequenced with the Illumina HiSeq 2500 platform with 100 bp paired end reads.

Raw FastQ reads were first analyzed using FastQC [41], and the FastP program was used for adapter removal and trimming of the sequences using the "-g" flag to remove poly $\mathrm{G}$ sequences and the "-detect_adapter for_pe" flag to remove paired end adapters [42]. Pair end reads were mapped to the Helicoverpa armigera genome (GCF_002156985.1) using HISAT2 v2.1.0 [43] with default parameters. The mapped reads were further sorted using Samtools v1.10-38 [44] and FeatureCounts v2.0.0 was used to quantify gene expression using the official gene set GFF file (https://data.csiro.au/dap/ landingpage?pid=csiro\%3A23812). All raw counts were normalized to transcripts per million (TPM) for cross sample comparisons.

\section{Nezara viridula maintenance}

The eggs of $N$. viridula were obtained from Bayer AG and reared in large mesh cages. Eggs, nymphs, and adults were kept under controlled conditions at $23 \pm$ $1{ }^{\circ} \mathrm{C}$ with a 12:12 light:dark photoperiod. The diet of nymphs and adult insects was the same: organic beans, carrots, as well as sunflower seeds.

\section{dsRNA synthesis}

cDNA from nymphs was used as a template for dsRNA synthesis. Amplicons of a 300-400 bp region of selected genes was amplified by PCR with Phusion ${ }^{\circ}$ DNA Polymerase Kit (New England BioLabs). The forward and the reverse primers had a T7 promoter sequence at their 5' end (Table S3). PCR conditions were $98{ }^{\circ} \mathrm{C}$ for $30 \mathrm{~s}$ for an initial denaturation, followed by 35 cycles of denaturation at $98{ }^{\circ} \mathrm{C}$ for $10 \mathrm{~s}$, annealing at $64{ }^{\circ} \mathrm{C}$ for $20 \mathrm{~s}$, extension at $72{ }^{\circ} \mathrm{C}$ for $20 \mathrm{~s}$, and a final step of extension at $72{ }^{\circ} \mathrm{C}$ for $5 \mathrm{~min}$. After PCR, the amplicons were purified using NucleoSpin ${ }^{\circ}$ Gel and PCR Clean-up kit (Macherey-Nagel). Purity was checked on $1.5 \%$ agarose gels. Purified DNA was then used as a template for in vitro dsRNA synthesis using the HiScribe ${ }^{\mathrm{Tm}}$ T7 High Yield RNA Synthesis Kit (New England BioLabs). Extraction (Phenol:Chloroform) and ethanol precipitation was performed according to the manufacturers protocol (New England BioLabs). The resulting dsRNA was diluted in injection buffer at a final concentration of $2 \mu \mathrm{g} /$ $\mu \mathrm{L}$.

\section{Nezara nymph injection}

Nymph injections were performed using a protocol established in a previous publication [45]. Three days after hatching, nymphs were transferred to agar step block that had been incubated on ice for $10 \mathrm{~min}$. Nymphs were lined up with their dorsal side facing the agar and, were incubated $\pm 5 \mathrm{~min}$ on ice. Approximately, $20 \mathrm{~nL}$ of $2 \mu \mathrm{g} / \mu \mathrm{L}$ dsRNA was injected to each nymph using an IM 300 Microinjector (Narishige, Japan). In total, eight different genes were tested, and ds-LacZ was used as a negative control. The injected nymphs were transferred to separate boxes under laboratory conditions and mortality was observed by the naked eye between 3 and 10 days after injection. Mortality was 
measured by Schneider-Orelli's formula. The experiment was performed with 75 nymphs in each repetition. Of these 25 nymphs ( 5 replicates of 5 nymphs) were collected for RNA extraction 5 days after injection.

For qPCR, TriZol was used to extract RNA of 5 nymphs, of which $1 \mu \mathrm{g}$ was used for cDNA synthesis using the oligo-dT primer and the reverse transcriptase kit from EnzyQuest (Heraklion, Greece). SYBRGreen master mix (Invitrogen) was then used for quantification with primers specific to each target gene or the RP60 and 18 s housekeeping genes (Table S3) by employing the $\Delta \Delta \mathrm{Ct}$ method. All reactions were set up with $.5 \mu \mathrm{M}$ of each primer and run on a CFX Connect (BioRad) machine. Efficiency estimates for all primer pairs were obtained with a 5 -fold dilution series, and at least 3 biological replicates were performed for each gene.

\section{Data availability}

The full source code for the ABC_scan pipeline is available on GitHub (https://github.com/shanedenecke/ABC_ scan.git), and a web application for users to identify $\mathrm{ABC}$ transporters from fasta protein files is available at (http://chrysalida.imbb.forth.gr:3838/ABC_scan/). The full analysis for this study (including BUSCO, CAFE etc.) is also available on GitHub (https://github.com/ shanedenecke/ABC_ID_SCRIPTS.git). Raw RNA-seq data from $\mathrm{H}$. armigera were submitted to the sequence read archive (SRA) under the bioproject accession (PRJNA719695).

\section{Results}

Annotation of $A B C$ transporters across arthropod species In order to understand and study the evolution of the $A B C$ transporter superfamily across arthropods, we designed the $A B C \_s c a n$ pipeline to identify and classify $\mathrm{ABCs}$ in 193 non-model species (excluding the 4 model species used in the search algorithm) with sequenced genomes derived from sources such as OrthoDB, NCBI, and others. Of these, 158 had BUSCO single copy completeness scores of $>80 \%$ and were analyzed with the $A B C \_$scan pipeline (Table S1). A total of 8,803 predicted total transporter sequences were identified averaging $55 /$ species, ranging from a low of 34 in the green orchard bee Euglossa dilemma to a high of 132 in the springtail Folsomia candida (Figure S1, Table S1). The general quality of the $A B C$ sscan identification algorithm was assessed by comparing the numbers of predicted transporters against numbers previously reported in the literature. Discrepancies between the $A B C \_s c a n$ pipeline implemented in this study and literature derived predicted transporters ranged from a $7 \%$ overestimation to an $8 \%$ underestimation with a mean deviation of $0.3 \%$ (Figure S2; Table S4), suggesting that the $A B C \_s c a n$ pipeline was generally suitable for predicting $A B C$ gene sets across arthropods. A publicly facing web application was also developed using R-shiny where users can upload a predicted protein set for a species (fasta format), which can then be scanned for $\mathrm{ABC}$ transporters (http:// chrysalida.imbb.forth.gr:3838/ABC_scan/).

A preliminary understanding of how $\mathrm{ABC}$ families differed among arthropod lineages was gained by dividing species into taxonomic groups and comparing their family sizes. Small amounts of variation were observed among the $\mathrm{ABC}-\mathrm{BH}, \mathrm{ABC}-\mathrm{D}, \mathrm{ABC}-\mathrm{E}$, and $\mathrm{ABC}-\mathrm{F}$ families (Figure S3; Table S5). While there were statistical differences among the ABC-D family, the small magnitude of the change made it difficult to explore in detail. The largest differences from the Kruskal-Wallis test comparisons were concentrated in the $\mathrm{ABC}-\mathrm{A}, \mathrm{ABC}-\mathrm{BF}$, $\mathrm{ABC}-\mathrm{C}, \mathrm{ABC}-\mathrm{G}$, and $\mathrm{ABC}-\mathrm{H}$ families (Fig. 2; Table S5). The ABC-G family was notable in that it appeared much smaller in all Arachnids (median of 3.5 genes) versus a median of 15 genes for all species combined. The arachnid T. urticae is an extreme outlier with 23 ABCG genes. The ABC-C family was significantly higher in Coleoptera with a median of 27 genes compared to 13 found among all arthropods. This expansion appeared to be specific to the Cucujiformia species such as T. castaneum and Leptinotarsa decemlineata (Figure S4). However, the most striking expansions supported by the greatest number of species were among lepidopteran $\mathrm{ABC}-\mathrm{B}$ full transporters and hemipteran $\mathrm{ABC}-\mathrm{H}$ transporters (Fig. 2). Therefore, these two cases were explored in further detail.

\section{ABC-B Full Transporters in Lepidoptera}

The ABC-B family is known to play roles in physiological homeostasis and drug transport. To study the evolutionary history of the Lepidoptera expansions, we built phylogenetic trees at the species and gene level. The Lepidoptera expansion of ABC-BF transporters appeared to take place in all members of this clade, and CAFE analysis did not identify any significant expansions or contractions within the Lepidoptera clade (Fig. 3A). Further characterization of the $\mathrm{ABC}-\mathrm{BF}$ expansion was performed by creating a phylogeny of all ABC-BF transporters from 6 Lepidoptera species and $N$. viridula, which is not a Lepidoptera but has a large number of ABC-BF transporters. Among the Lepidoptera there was no observed species specific clustering of transporters while all $N$. viridula $\mathrm{ABC}-\mathrm{BF}$ transporters grouped together (Fig. 3B). This suggests that the ABC-BF expansion in Lepidoptera likely occurred at the beginning of the Lepidoptera lineage.

Transcriptomic analysis on the midgut, Malpighian tubules, and central nervous system of the model 


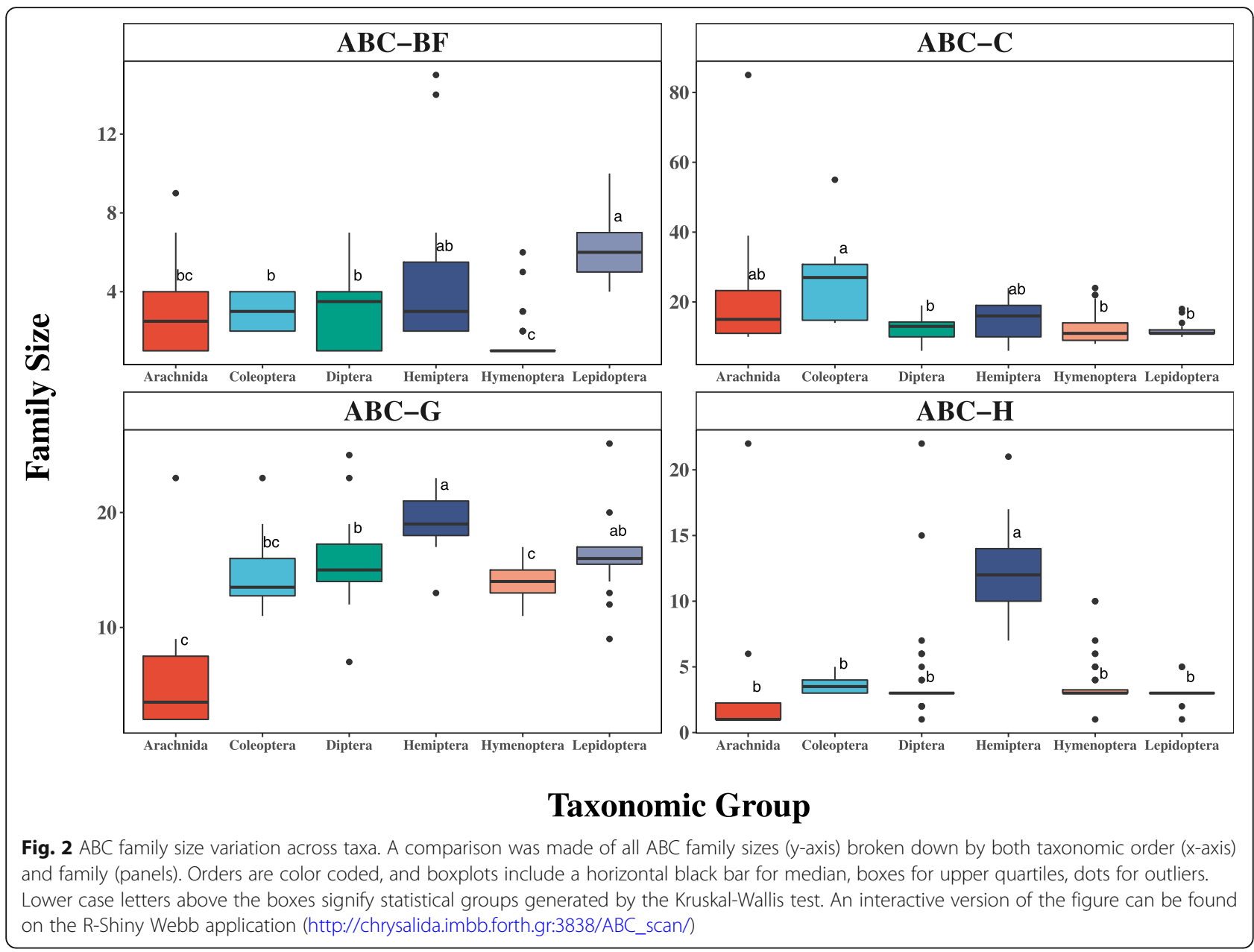

Lepidoptera $H$. armigera was employed to further characterize the ABCB full transporters. 7 full transporters were identified in our pipeline which were in agreement with a previous study and were named in accordance with the official gene set nomenclature (Fig. 4; Table S6) [46]. In the Malpighian tubules several paralogues showed noteworthy expression measured in transcripts per million (TPM) including HaABC-B3 (761.85 TPM), HaABC-B1 (131.68 ТРM), HaABC-B7 (116.46 TPM), HarmABC-B2 (31.24 TPM). Interestingly, in the midgut tissue only one gene, $H a A B C-B 7$ (89.34 TPM) was highly expressed. Similarly, the central nervous system expressed only $H a A B C-B 1$ (44.62 TPM) at high levels. The paralogues $H a A B C-B 5, H a A B C-B 6, H a A B C-B 11$ all showed minimal expression in all the tissues sampled. In total, the variable expression indicates that different P-glycoprotein paralogues show highly divergent expression patterns and that each epithelial tissue sampled has a different paralogue that predominates.

\section{The ABC-H Family in Hemiptera}

Next, we considered the higher number of $\mathrm{ABC}-\mathrm{H}$ transporters, which have a less defined physiological role. While CAFE found an elevated level of ABC-H transporters in Hemiptera compared to other arthropods, it also suggested that the lineage containing aphids and whiteflies (Sternorrhyncha such as M. persicae, A. pisum and $B$. tabaci) and the branch containing other Hemiptera (stink bugs, planthoppers etc. such as $N$. viridula, $R$. prolixus, and $N$. lugens), may have underwent additional expansions specific to each lineage (Fig. 5A). To address this question, a phylogenetic tree was generated from all ABC-H genes from 6 Hemiptera along with T. urticae and $D$. pulex as outgroups. A subset of these $\mathrm{ABC}-\mathrm{H}$ transporters orthologous to the Drosophila gene Snu clustered as 1:1 orthologues as reported previously [6]. However, the $\mathrm{ABC}-\mathrm{H}$ proteins from $D$. pulex and $T$. urticae grouped completely independently, suggesting independent expansions of the $\mathrm{ABC}-\mathrm{H}$ family in each of these two species. Likewise, most non-Snu ABC-H 


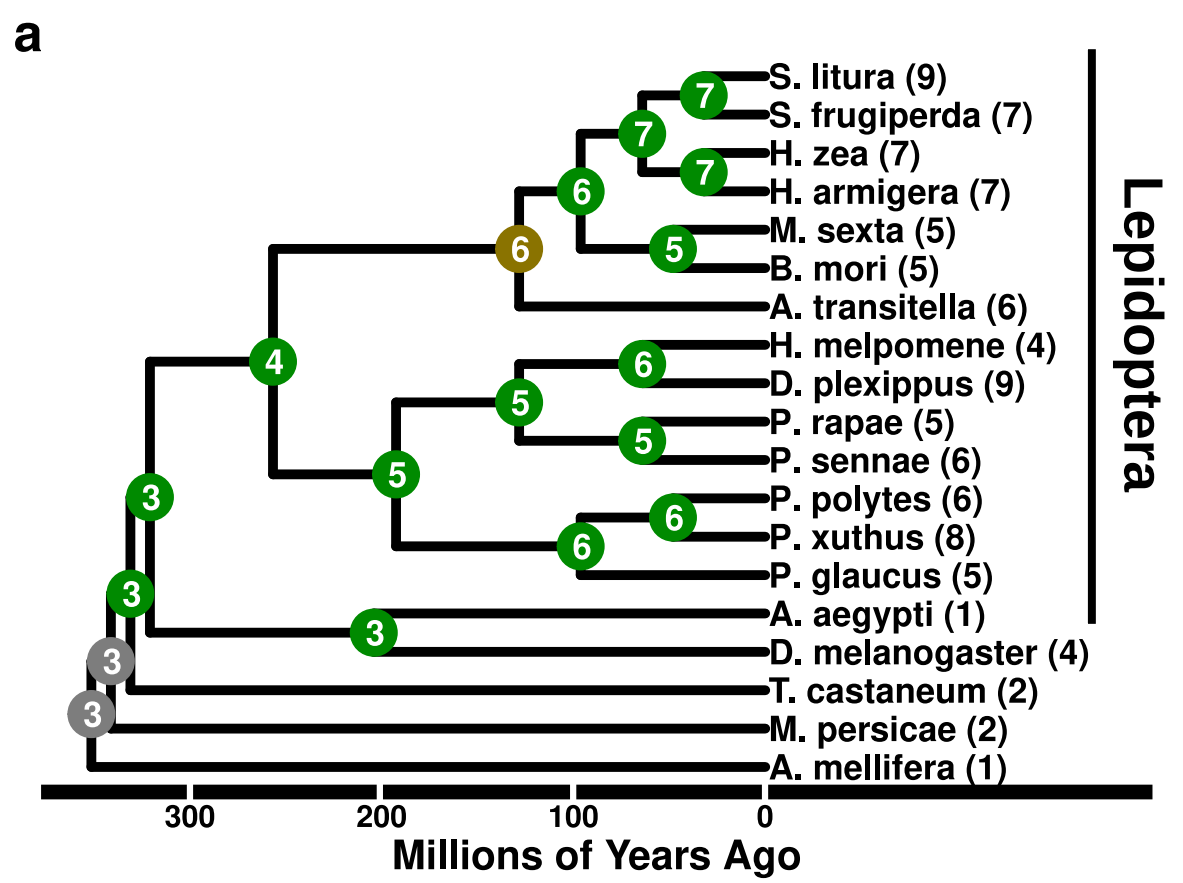

b

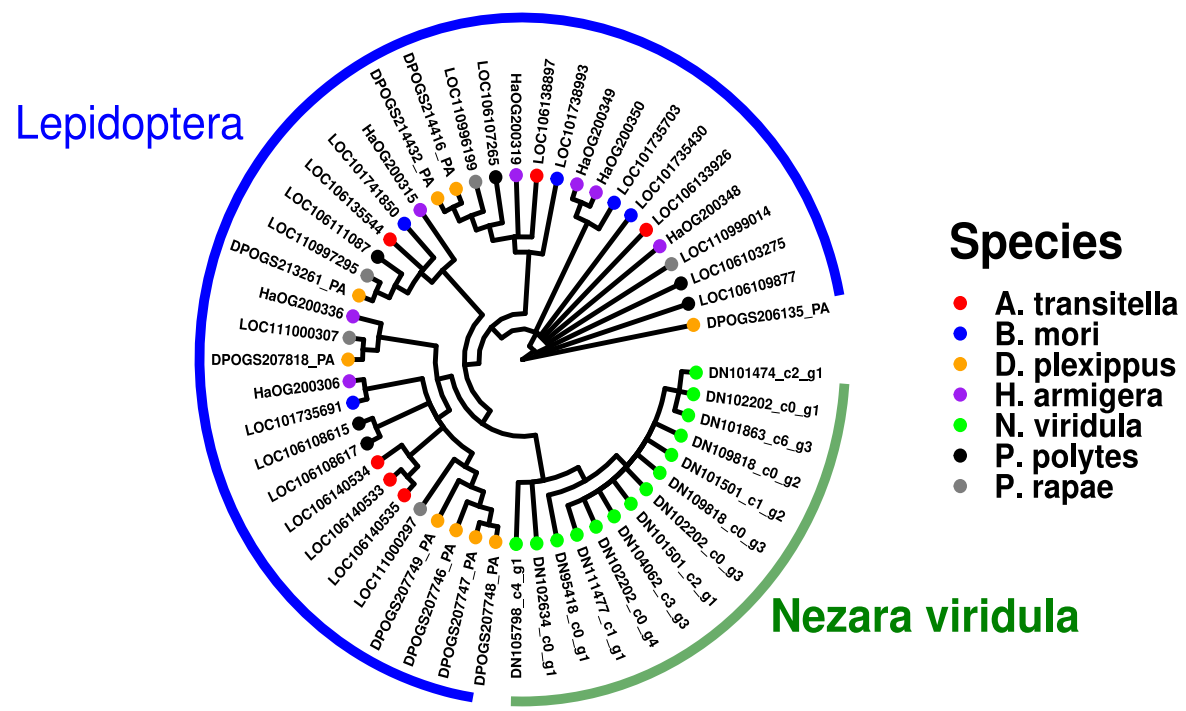

Fig. 3 The ABC- B full transporters in Lepidoptera. a The evolution within the ABC-B full transporter family was analyzed with CAFE. Each tip of the tree represents a species mostly of Lepidoptera. The numbers present next to each tip correspond to the number of predicted ABC- B full transporters, while the node numbers correspond to CAFE predictions for ABC-B full transporter family sizes. Color coding of the nodes signifies bootstrap support with $>90 \%=$ Green; $70-90 \%=$ Yellow; $<70 \%=$ Red; NA = Gray. b A phylogeny of a subset of ABC- B full transporters was made in order to understand the relationship of these transporters among Lepidoptera and other groups. The majority of P-glycoproteins from Lepidoptera do not show species specific expansions suggesting that an expansion occurred common to all Lepidoptera. In contrast, the clustering of the N. viridula P-glycoproteins suggest a distinct origin for this expansion

transporters from Sternorrhyncha (aphids and whiteflies such as $M$. persicae and D. vitifoliae) clustered together and formed a sister group to the majority of other Hemiptera genes which formed an almost completely monophyletic cluster of ABC-H transporters (Fig. 5B). The independent grouping of Sternorrhyncha and nonSternorrhyncha $\mathrm{ABC}-\mathrm{H}$ genes suggests that while the $\mathrm{ABC}-\mathrm{H}$ family is larger in all Hemiptera, it may have undergone multiple expansions at different times in the evolution of this clade. 


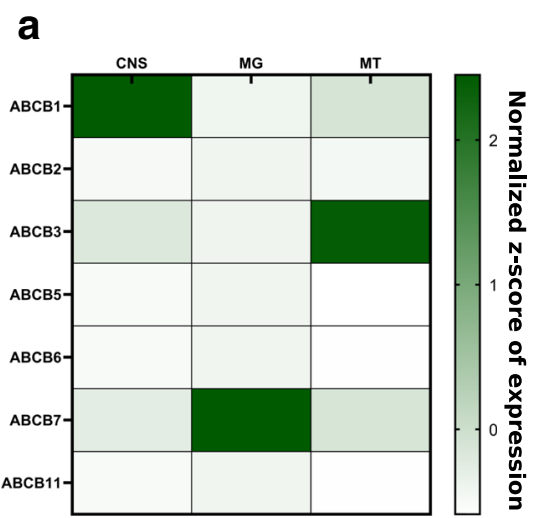

\section{b}

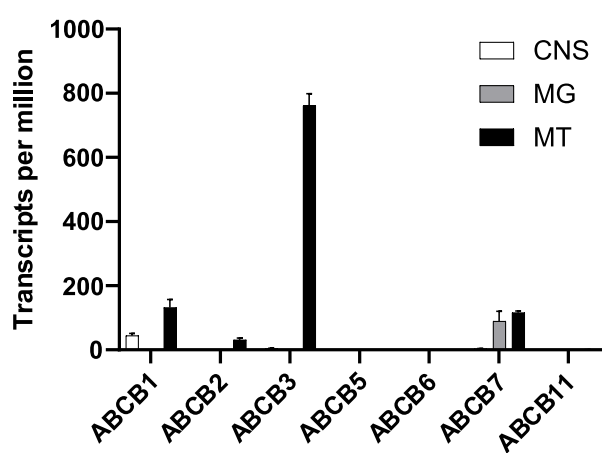

Fig. 4 Expression of the ABC-B full transporters in. H. armigera. a Heatmap showing differential expression of the ABC-B paralogues in different tissues of fifth larval stage $\mathrm{H}$. armigera based on transcript per million values, TPM (Z-transformed) values obtained using RNA-seq. Where CNS = central nervous system, $M G=$ midgut and $M T=$ Malpighian tubules. Each box represents the mean value of four biological replicates $(n=4)$. $\mathbf{b}$ Bar graph showing the total expression of each $\mathrm{ABC}-\mathrm{BF}$ gene in each tissue (CNS = white, $M G=$ grey, $M T=$ black $)$

To study the potential roles of hemipteran specific $\mathrm{ABC}-\mathrm{H}$ transporters, we used RNAi to individually knock down the expression of several $\mathrm{ABC}-\mathrm{H}$ genes in the stink bug $N$. viridula. Using previous transcriptomic information [47], it was observed that the $\mathrm{ABC}-\mathrm{H}$ genes were expressed primarily in the M4 midgut region and non-gut tissue (Table S7). We selected 8 out of 14 genes for RNAi knockdown based on high expression levels. Significant reduction in expression levels ranging from 70 to $97 \%$ was observed for 5 out of the 8 genes following knockdown attempts (Figure S5). No observable phenotype was detected in knockdown individuals apart from the constructs targeting the gene homologous to D. melanogaster Snu (DN106392_c1_g2), which led to mortality in almost all nymphs 10 days post injection (Fig. 6). A shriveling of the body was observed in these nymphs suggesting that desiccation may underpin this mortality. The Snu gene is highly conserved appearing as a 1:1 orthologue in all Hemiptera species examined and it is closely related to the $S n u$ gene previously studied in other insects (see discussion).

\section{Discussion}

There has been substantial interest in the ABC superfamily among arthropods both as mediators of pesticide resistance and as targets for pest control. Their status as one of the largest and best understood gene superfamilies also make them a highly interesting evolutionary model. The increased rate of genome and transcriptome sequencing in recent years has made it possible to observe how such superfamilies evolve on various evolutionary timescales using comparative genomics approaches. While several studies have performed annotations in individual species or looked at closely related species (See references in Table S4), there has been few comparative genomic studies of $\mathrm{ABC}$ evolution across arthropods. The two exceptions were Dermauw et al. [6], which used a small number of species and Rane et al. [28] which, only looked at total $A B C$ number rather than specific family evolution.

\section{The ABC_scan pipeline}

In this study, we implemented an algorithm to identify $\mathrm{ABC}$ transporters from the predicted protein set of nonmodel arthropods. The algorithm performs well $(<10 \%$ error) when benchmarked against manually curated datasets in other species (Figure S2), and it appears that most differences centered around different starting protein sets and our decision to remove short amino acid fragments under 250 residues. Because $A B C \_s c a n$ represented a reasonably robust tool to catalogue and sort $\mathrm{ABC}$ transporters, a standalone executable version of ABC_scan (https://github.com/shanedenecke/ABC_scan. git) and web application (http://chrysalida.imbb.forth. gr:3838/ABC_scan/) were designed to annotate new protein sets. This appears to be of interest as there are a substantial number of studies that are composed almost exclusively of such annotations with often less comprehensive identification pipelines. As the pipeline is generic apart from the use of several arthropod specific $\mathrm{ABC}$ sets in order to sort candidate transporters, it could theoretically be adapted to non-arthropod species, although this was not tested in this study.

However, there are several limitations to $A B C_{-}$scan. Because it searches amino acid sequences, poor genome annotations will under or overestimate $\mathrm{ABC}$ gene counts (hence the BUSCO threshold used here). Furthermore, manual curation will almost always be able to provide 


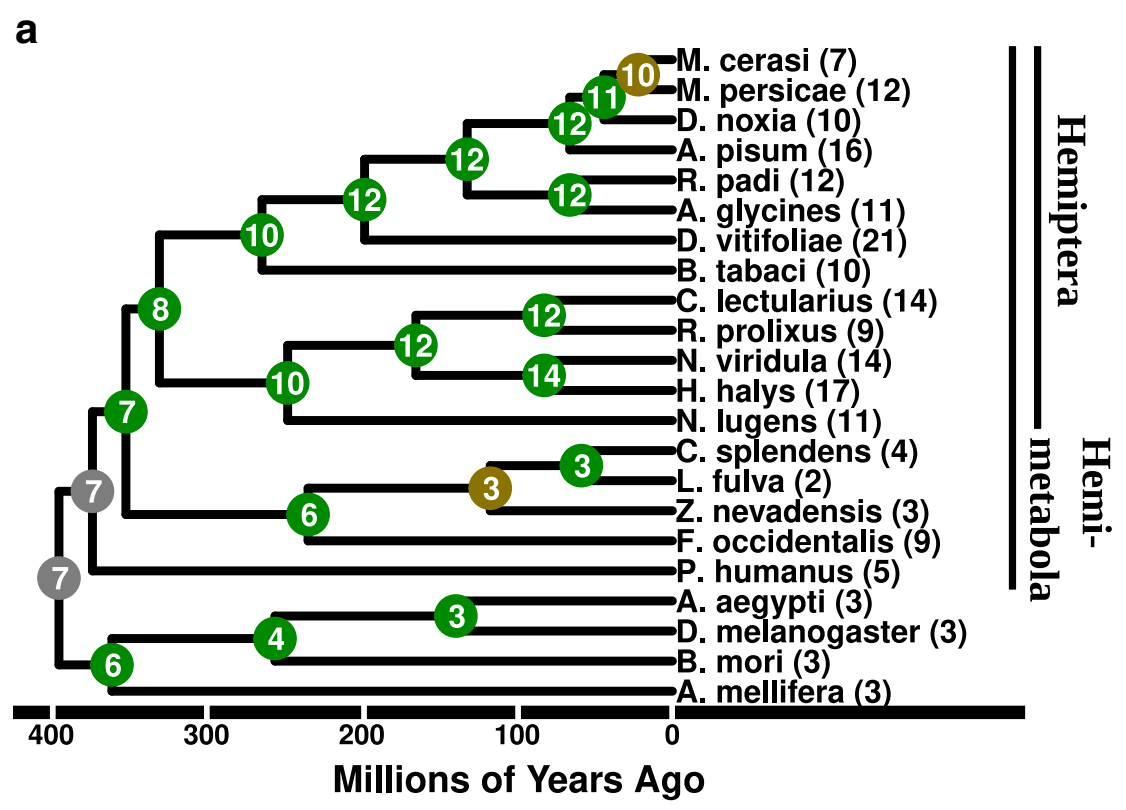

b

Daphnia

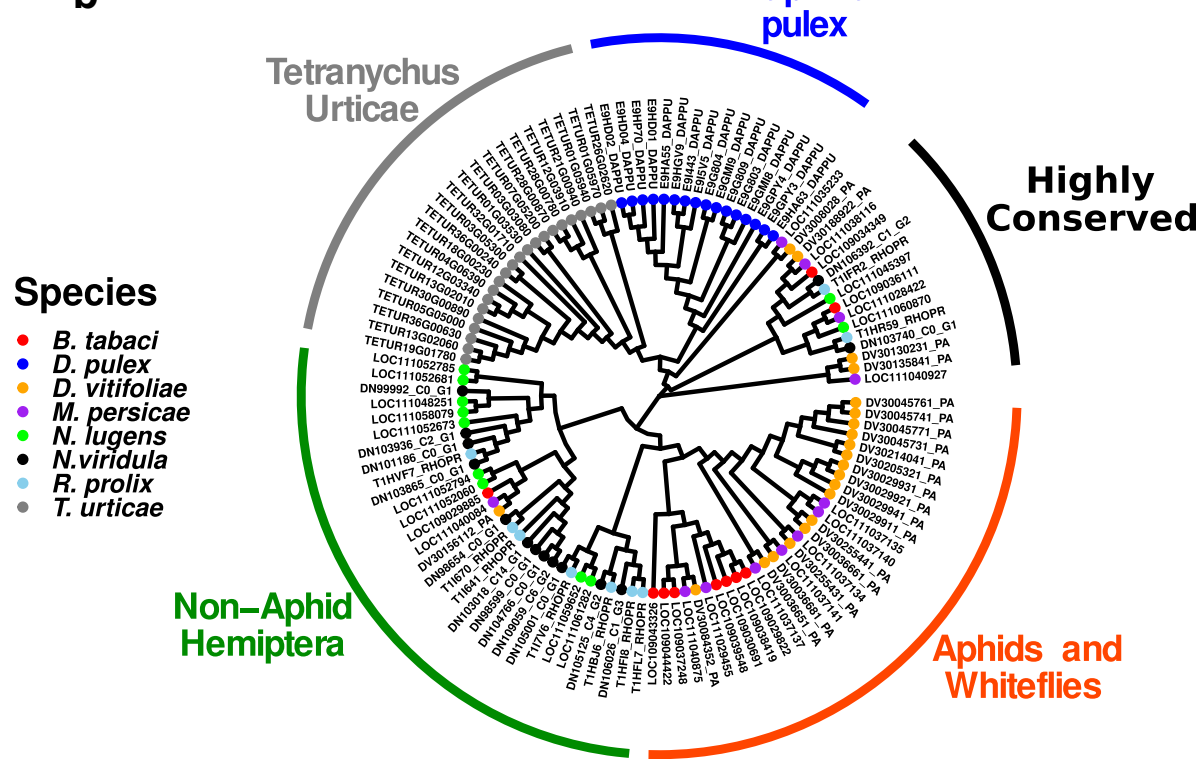

Fig. 5 ABC-H family phylogeny in Hemiptera. a The evolution within the ABC-H transporter family was analyzed with CAFE. Each tip of the tree represents a species mostly of Hemiptera. The numbers present next to each tip correspond to the number of predicted ABC-H transporters, while the node numbers correspond to CAFE predictions for family sizes. Color coding of the nodes signifies bootstrap support with $>90 \%=$ Green; 70-90\% =Yellow; $\angle 70 \%=$ Red; NA = Gray. $\mathbf{b}$ A phylogeny of a subset of ABC-H transporters was made so as to understand the relationship of these transporters among Hemiptera and other groups. ABC-H proteins appear to group based on lineage, suggesting that multiple expansions occurred during the evolution of Arthropods

more reliable estimates and fine tune gene models. Still, the accessibility of $A B C \_$scan and the fact that it is more comprehensive than many published methodologies suggests that it might be of use to the wider research community occupying a space between detailed manual curation and completely automated annotations from larger databases (e.g. NCBI).
The expansion of xenobiotic transporting genes in Coleoptera and Lepidoptera

Deploying $A B C \_s c a n$ to search 158 non-model arthropods identified several interesting evolutionary trends in the $\mathrm{ABC}-\mathrm{C}$ and $\mathrm{ABC}-\mathrm{BF}$ families previously associated with xenobiotic transport. The ABC-C family was not the subject of intense focus in this study but showed a 


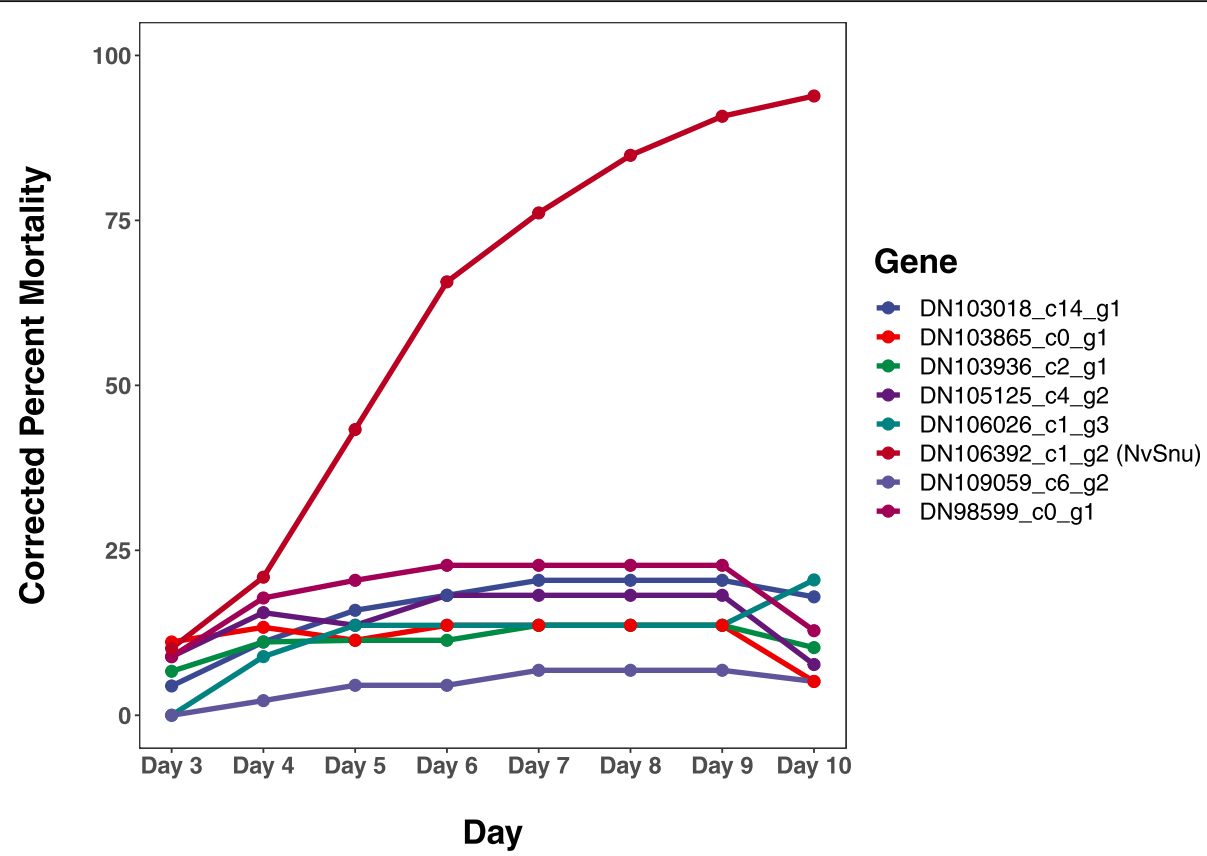

Fig. 6 RNAi lethality of $A B C-H$ transporters. The essentiality of the ABC-H transporters was examined in $N$. viridula using RNAi. Following dsRNA injection, mortality was measured from 3 to 10 days. The $x$ axis represents time in days, and the $y$-axis represents mortality corrected to dsLacZ injected individuals. Only the Snu gene gave substantial amounts of mortality, while all other ABC-H genes displayed mortality $<30 \%$

clear expansion in polyphagous beetles (Cucujiformia) compared to other beetles and non-beetle insects (Figure S4). A recent study implicated members of this ABC-C expansion in pesticide resistance, which highlights the adaptive potential of recent gene family expansions [48]. A significant expansion of the $\mathrm{ABC}-\mathrm{BF}$ in Lepidoptera was also detected and seems to have occurred soon after the divergence of this order (Fig. 5). This expansion runs in parallel to a similar expansion of the SLC22 (organic ion) transporter family in this group, which has also been associated with xenobiotic transport [27]. The expansion of these "detoxification" families within insect lineages may also suggest adaptations to a more complex diet of plant materials, but functional work will be needed to confirm or reject this hypothesis and implicate individual genes.

Characterization of the expression patterns of the $\mathrm{ABCB}$ full transporter family in $H$. armigera yielded interesting results. Despite residing in a genomic cluster, $H a A B C-B 1, H a A B C-B 2$ and $H a A B C-B 3$ showed relatively divergent expression patterns (Fig. 4). A Similar situation was observed in the leaf beetle Chrysochus auratus where three P-glycoprotein paralogues at the same genomic locus display strong differences in their expression patterns [49]. The paralogues in D. melanogaster also show such partitioning among its four P-gp paralogues [15]. In the $H$. armigera central nervous system the $H a A B C-B 1$ gene predominated, and it is likely this localizes to the blood brain barrier, acting as a xenobiotic transporter similar to the case in other insects and humans [12, 50]. Similarly, the midgut expressed only one transporter, $H a A B C-B 7$, which also showed abundant expression in the Malpighian tubules (Table S6). In other insects such as Plutella xylostella, L. decemlineata and Trichoplusia ni the expression of P-gp orthologues was enriched in the midgut and Malpighian tubules [5153], and similar results were also obtained from a study of $H$. armigera transporters with qPCR [54]. Further work will be needed to fully understand the relationship between expression patterns of P-glycoprotein paralogues within and between species. Additional functional work is also necessary. A recent study showed that CRISPR-Cas9 mediated knockout of another Lepidoptera Spodoptera exigua increased sensitivity to emamectin benzoate [55]. Of all the Pglycoproteins in $H$. armigera, $H a A B C-B 7$ has underwent functional characterization as a purified protein [56], and a recent CRISPR mediated knockout of $H a A B C-B 6$ increased sensitivity to the plant secondary metabolite gossypol [57].

\section{RNAi knockdown of ABC-H transporters in N. viridula}

The discovery of an enrichment of $\mathrm{ABC}-\mathrm{H}$ transporters in Hemiptera prompted us to examine what physiological roles these transporters play. To the authors knowledge, this study is the first attempt to study $\mathrm{ABC}$ $\mathrm{H}$ genes in Hemiptera. From the phylogenetic analysis it appears that there were independent expansions of 
ABC-H transporters among Sternorrhyncha (aphids and whiteflies) and non-Sternorrhyncha, along with a more conserved cluster of 1:1 orthologues (Fig. 5B). Many of these transporters were expressed in the M4 region which is divergent from the rest of the midgut and is thought to play a role in harboring symbiotic microorganisms [58]. However, the functional role these transporters play in this tissue is not clear.

Knockdown of the more conserved DN106392_C1_G2, induced a high level of mortality and nymphs appeared to desiccate or die during the transition from the 2nd to the 3rd nymphal stage (Fig. 6). This gene is also the most closely related to $\mathrm{Snu}$, which has been characterized as an essential gene in cuticular formation through hydrocarbon transport at the epidermis [20-22]. In contrast, no observable phenotype was detected when the hemipteran specific $\mathrm{ABC}-\mathrm{H}$ genes were targeted with dsRNA despite a successful knockdown. While this may be due to the technical limitations of RNAi, one explanation may be that the enrichment of $\mathrm{ABC}-\mathrm{H}$ transporters in Hemiptera has resulted in functional redundancy between the transporters of this family. The evolutionary reason for this gene expansion in Hemiptera will require in-depth molecular studies of the physiological role of these so far largely unexplored $\mathrm{ABC}-\mathrm{H}$ transporter family.

\section{Supplementary Information}

The online version contains supplementary material available at https://doi. org/10.1186/s12864-021-07861-2.

Additional file 1: Figure S1. The total number of $A B C$ transporters in each species ( $x$-axis) was calculated for each species included in the analysis.

Additional file 2: Figure S2. The predicted numbers of $A B C$ transporters in the ABC_scan pipeline were benchmarked against previously published $A B C$ transporter datasets. The \% difference in total number of $A B C$ transporters ( $y$-axis) was plotted for each species ( $x$-axis). For all species tested, the difference was less than 10\% (dotted line).

Additional file 3: Figure S3. A comparison was made among the nonvariable $A B C$ families ( $A B C-B H, A B C-D, A B C-E$, and $A B C-F$ ) with family size (y-axis) broken down by both taxonomic order (x-axis) and family (panels). Orders are color coded, and boxplots include a horizontal black bar for median, boxes for upper quartiles, dots for outliers. Lower case letters above the boxes signify statistical groups generated by the KruskalWallis test.

Additional file 4: Figure S4. A CAFE analysis for ABC-C proteins was performed with a focus on Coleoptera. Higher numbers of ABC-C proteins were observed in Cucujiformia beetles (e.g. T. castaneum, L. decemlineata) compared to other beetles and non-beetle arthropods. The numbers present next to each tip correspond to the number of predicted $A B C-C$ transporters, while the node numbers correspond to CAFE predictions for $A B C-B$ full transporter family sizes. Color coding of the nodes signifies bootstrap support with $>90 \%$ percent $=$ Green; $70-90 \%=$ Yellow; $<$ $70 \%=$ Red; NA=Gray.

Additional file 5: Figure S5. qPCR was performed for all ABC-H genes knocked down in $N$. viridula. For each gene at least 3 biological replicates were performed, and all values were calculated using the $\Delta \Delta \mathrm{Ct}$ method and normalized to 1 .
Additional file 6: Table S1. This table shows all the metadata associated with every species included in the analysis. $A B C$ counts are missing for species with a BUSCO scores of below \%80 Single copy orthologues.

Additional file 7: Table S2. Species level phylogenies used in CAFE were constructed with the species shown in this table. Outgroups are highlighted in yellow.

Additional file 8: Table S3. All primers used in the N. viridula RNAi screen are shown.

Additional file 9: Table S4. The raw data for Figure $\mathrm{S} 2$ is shown, which contains the total number of $\mathrm{ABC}$ transporters identified in $A B C$ _scan and the literature for a subset of species. Also included is the source of the literature derived counts.

Additional file 10: Table S5. All significant outputs of the Kruskal-Wallis analysis measuring the effect of taxonomic group on $A B C$ family size are listed.

Additional file 11: Table S6. A transcriptomic analysis of $H$. armigera midgut, Malpighian tubules and central nervous system was undertaken to examine P-glycoprotein expression in these epithelial tissues. H. armigera genes are listed with their official gene set (OGS) codes, corresponding NCBI accession numbers, and their expression values in each tissue displayed in transcripts per million (TPM).

Additional file 12: Table S7. All predicted $A B C-H$ genes in $\mathrm{N}$. viridula are listed along with transcriptomic data derived from [47].

\section{Acknowledgements}

The authors would like to thank Christen Baden of Bayer Crop Sciences for providing Nezara viridula eggs on multiple occasions. Furthermore, the authors would like to thank Panagiotis loannidis for being a useful resource for technical troubleshooting of computational issues. Lastly, the authors would like to thank loannis Livadaras for assistance with N. viridula injections.

\section{Authors' contributions}

SD wrote the manuscript and performed bioinformatic analysis. IV and OD performed experimental work. MK analyzed the data and wrote sections of the manuscript. NGHL developed the web application. BB, RN, SG and JV provided significant input into the writing of the manuscript and assisted in study design. The author(s) read and approved the final manuscript.

\section{Funding}

Ivan Rankić was funded via an ERASMUS Trainee Internship Program involving the Mendel University (Brno45) and the Institute of Molecular Biology and Biotechnology (IMBB; Heraklion). Megha Kalsi, Ngoc Bao Hang Luong, and Shane Denecke are funded as a part of a joint collaboration between IMBB and Bayer AG.

\section{Availability of data and materials}

All data analyzed in this study is publicly available. All analysis scripts can be found on GitHub https://github.com/shanedenecke/ABC_scan.git. The data can also be accessed via a web application at http://chrysalida.imbb.forth.gr:3 838/ABC_scan/. Raw sequencing can be found on the sequence read archive at PRJNA719695.

\section{Declarations}

Ethics approval and consent to participate

Not applicable to this study.

\section{Consent for publication}

All authors have consented to the publication of this manuscript.

\section{Competing interests}

Megha Kalsi, Ngoc Bao Hang Luong, and Shane Denecke are funded as a part of a joint collaboration between IMBB and Bayer AG. Sven Geibel, Ralf Nauen, Benjamin Buer are employees at Bayer Crop Sciences. All other authors have no competing interests. 


\section{Author details}

${ }^{1}$ Institute of Molecular Biology \& Biotechnology, Foundation for Research \& Technology Hellas, 100 N. Plastira Street, 700 13, Heraklion Crete, Greece. ${ }^{2}$ Department of Chemistry and Biochemistry, Mendel University in Brno, Zemedelska 1, 61300 Brno, Czechia. ${ }^{3}$ CropScience Division, Bayer AG, R\&D Pest Control, D-40789 Monheim, Germany. ${ }^{4}$ Laboratory of Pesticide Science, Department of Crop Science, Agricultural University of Athens, Athens, Greece.

Received: 15 April 2021 Accepted: 23 June 2021

Published online: 19 July 2021

\section{References}

1. Theodoulou FL, Kerr ID. ABC transporter research: Going strong 40 years on. Biochem Soc Trans. 2015;43:1033-40. https://doi.org/10.1042/BST20150139.

2. Dassa E. Natural history of ABC systems: Not only transporters. Essays Biochem. 2011;50:19-42. https://doi.org/10.1042/BSE0500019.

3. Higgins CF. ABC transporters: from microorganisms to man. Annu Rev Cell Biol. 1992;8:67-113. https://doi.org/10.1146/annurev.cb.08.110192.000435.

4. Saier MH, Reddy VS, Moreno-Hagelsieb G, Hendargo KJ, Zhang Y, Iddamsetty V, et al. The transporter classification database (TCDB): 2021 update. Nucleic Acids Res. 2021;49:D461-7. https://doi.org/10.1093/nar/gkaa1 004.

5. Thomas GWC, Dohmen E, Hughes DST, Murali SC, Poelchau M, Glastad K, et al. Gene content evolution in the arthropods. Genome Biol. 2020;21:15. https://doi.org/10.1186/s13059-019-1925-7.

6. Dermauw W, Van Leeuwen T. The ABC gene family in arthropods: comparative genomics and role in insecticide transport and resistance. Insect Biochem Mol Biol. 2014;45:89-110. https://doi.org/10.1016/j.ibmb.2 013.11.001.

7. Merzendorfer H. ABC Transporters and Their Role in Protecting Insects from Pesticides and Their Metabolites. Elsevier; 2014. https://doi.org/10.1016/ B978-0-12-417010-0.00001-X.

8. Kalsi M, Palli SR. Cap n collar transcription factor regulates multiple genes coding for proteins involved in insecticide detoxification in the red flour beetle, Tribolium castaneum. Insect Biochem Mol Biol. 2017. https://doi.org/1 0.1016/j.ibmb.2017.09.009.

9. Adang MJ, Crickmore N, Jurat-Fuentes JL. Diversity of Bacillus thuringiensis Crystal Toxins and Mechanism of Action. 2014:39-87. https://doi.org/10.101 6/B978-0-12-800197-4.00002-6.

10. Hodges LM, Markova SM, Chinn LW, Gow JM, Kroetz DL, Klein TE, et al. Very important pharmacogene summary: ABCB1 (MDR1, P-glycoprotein). Pharmacogenet Genomics. 2011;21:152-61. https://doi.org/10.1097/FPC. 0b013e3283385a1c.

11. Sreeramulu K, Liu R, Sharom FJ. Interaction of insecticides with mammalian P-glycoprotein and their effect on its transport function. Biochim Biophys Acta - Biomembr. 2007:1750-7.

12. Lanning CL, Fine RL, Corcoran JJ, Ayad HM, Rose RL, Abou-Donia MB. Tobacco budworm P-glycoprotein: biochemical characterization and its involvement in pesticide resistance. Biochim Biophys Acta - Gen Subj. 1996; 1291:155-62. https://doi.org/10.1016/0304-4165(96)00060-8.

13. Luo L, Sun Y-J, Wu Y-J. Abamectin resistance in Drosophila is related to increased expression of P-glycoprotein via the dEGFR and dAkt pathways. Insect Biochem Mol Biol. 2013;43:627-34. https://doi.org/10.1016/j.ibmb.2 013.04.006.

14. Figueira-Mansur J, Ferreira-Pereira A, Mansur JF, Franco TA, Alvarenga ESL, Sorgine MHF, et al. Silencing of P-glycoprotein increases mortality in temephos-treated Aedes aegypti larvae. Insect Mol Biol. 2013;22:648-58. https://doi.org/10.1111/imb.12052.

15. Denecke S, Fusetto R, Batterham P. Describing the role of Drosophila melanogaster $\mathrm{ABC}$ transporters in insecticide biology using CRISPR-Cas9 knockouts. Insect Biochem Mol Biol. 2017;91:1-9. https://doi.org/10.1016/j. ibmb.2017.09.017.

16. Sturm $A$, Cunningham $P$, Dean $M$. The $A B C$ transporter gene family of Daphnia pulex. BMC Genomics. 2009:1-18.

17. Tian L, Song T, He R, Zeng Y, Xie W, Wu Q, et al. Genome-wide analysis of ATP-binding cassette (ABC) transporters in the sweetpotato whitefly, Bemisia tabaci. BMC Genomics. 2017;18:330. https://doi.org/10.1186/s12864-017-3 706-6.

18. Liu X-Q, Jiang H-B, Xiong Y, Peng P, Li H-F, Yuan G-R, et al. Genome-wide identification of ATP-binding cassette transporters and expression profiles in the Asian citrus psyllid, Diaphorina citri, exposed to imidacloprid. Comp Biochem Physiol Part D Genomics Proteomics. 2019;30:305-11. https://doi. org/10.1016/J.CBD.2019.04.003.

19. Dermauw W, Wybouw N, Rombauts S, Menten B, Vontas J, Grbic M, et al. A link between host plant adaptation and pesticide resistance in the polyphagous spider mite Tetranychus urticae. Proc Natl Acad Sci U S A. 2013;110:E113-22. https://doi.org/10.1073/pnas.1213214110.

20. Zuber R, Norum M, Wang Y, Oehl K, Gehring N, Accardi D, et al. The ABC transporter Snu and the extracellular protein Snsl cooperate in the formation of the lipid-based inward and outward barrier in the skin of Drosophila. Eur J Cell Biol. 2017. https://doi.org/10.1016/J.EJCB.2017.12.003.

21. Guo Z, Kang S, Zhu X, Xia J, Wu Q, Wang S, et al. The novel ABC transporter $\mathrm{ABCH} 1$ is a potential target for RNAi-based insect pest control and resistance management. Sci Rep. 2015;5:13728. https://doi.org/10.1038/ srep13728.

22. Yu Z, Wang Y, Zhao X, Liu X, Ma E, Moussian B, et al. The ABC transporter ABCH-9 $C$ is needed for cuticle barrier construction in Locusta migratoria. Insect Biochem Mol Biol. 2017;87:90-9. https://doi.org/10.1016/j.ibmb.2017. 06.005 .

23. i5K Consortium. The i5K Initiative: Advancing Arthropod Genomics for Knowledge, Human Health, Agriculture, and the Environment. J Hered. 2013;104:595-600. https://doi.org/10.1093/jhered/est050.

24. Qi W, Ma X, He W, Chen W, Zou M, Gurr GM, et al. Characterization and expression profiling of ATP-binding cassette transporter genes in the diamondback moth, Plutella xylostella (L.). BMC Genomics. 2016;17:760. https://doi.org/10.1186/s12864-016-3096-1.

25. Figueira-Mansur J, Schrago CG, Salles TS, Alvarenga ESL, Vasconcellos BM, Melo ACA, et al. Phylogenetic analysis of the ATP-binding cassette proteins suggests a new $A B C$ protein subfamily I in Aedes aegypti (Diptera: Culicidae). BMC Genomics. 2020;21:463. https://doi.org/10.1186/s12864-02006873-8.

26. Lane TS, Rempe CS, Davitt J, Staton ME, Peng Y, Soltis DE, et al. Diversity of $A B C$ transporter genes across the plant kingdom and their potential utility in biotechnology. BMC Biotechnol. 2016;16:47. https://doi.org/10.1186/s12 896-016-0277-6.

27. Denecke SM, Driva O, Luong HNB, loannidis P, Linka M, Nauen R, et al. The Identification and Evolutionary Trends of the Solute Carrier Superfamily in Arthropods. Genome Biol Evol. 2020;12:1429-39.

28. Rane RV, Ghodke AB, Hoffmann AA, Edwards OR, Walsh TK, Oakeshott JG. Detoxifying enzyme complements and host use phenotypes in 160 insect species. Curr Opin Insect Sci. 2019. https://doi.org/10.1016/J.COIS.2018.12. 008.

29. Waterhouse RM, Seppey M, Simão FA, Zdobnov EM. Using BUSCO to Assess Insect Genomic Resources. Insect Genomics. Hatfield; 2019;59-74. https:// doi.org/10.1007/978-1-4939-8775-7_6.

30. Eddy SR, Accelerated Profile HMM, Searches. Accelerated Profile HMM, Searches. PLoS Comput Biol. 2011;7:e1002195. https://doi.org/10.1371/journa I.pcbi.1002195.

31. Camacho C, Coulouris G, Avagyan V, Ma N, Papadopoulos J, Bealer K, et al. BLAST+: architecture and applications. BMC Bioinformatics. 2009;10:421. https://doi.org/10.1186/1471-2105-10-421.

32. Dean M, Hamon Y, Chimini G. The human ATP-binding cassette (ABC) transporter superfamily. J Lipid Res. 2001;42:1007-17. http://www.ncbi.nlm. nih.gov/pubmed/11441126. Accessed 24 Mar 2016.

33. Broehan $\mathrm{G}$, Kroeger $\mathrm{T}$, Lorenzen $\mathrm{M}$, Merzendorfer $\mathrm{H}$. Functional analysis of the ATP-binding cassette (ABC) transporter gene family of Tribolium castaneum. BMC Genomics. 2013;14:6. https://doi.org/10.1186/1471-2164-14-6.

34. Mendes FK, Vanderpool D, Fulton B, Hahn MW. CAFE 5 models variation in evolutionary rates among gene families. Bioinformatics. 2021;36:5516-8. https://doi.org/10.1093/bioinformatics/btaa1022.

35. Emms DM, Kelly S. OrthoFinder: Phylogenetic orthology inference for comparative genomics. Genome Biol. 2019;20:1-14.

36. Katoh K, Standley DM. MAFFT Multiple Sequence Alignment Software Version 7: Improvements in Performance and Usability. Mol Biol Evol. 2013; 30:772-80. https://doi.org/10.1093/molbev/mst010.

37. Capella-Gutiérrez S, Silla-Martínez JM, Gabaldón T. trimAl: a tool for automated alignment trimming in large-scale phylogenetic analyses. Bioinformatics. 2009; 25:1972-3. https://doi.org/10.1093/bioinformatics/btp348.

38. Kozlov AM, Darriba D, Flouri T, Morel B, Stamatakis A, Wren J. RAxML-NG: A fast, scalable and user-friendly tool for maximum likelihood phylogenetic inference. Bioinformatics. 2019:4453-5. 
39. Misof B, Liu S, Meusemann K, Peters RS, Donath A, Mayer C, et al. Phylogenomics resolves the timing and pattern of insect evolution. Science. 2014;346:763-7. https://doi.org/10.1126/science.1257570.

40. Yu G, Smith DK, Zhu H, Guan Y, Lam T-Y. ggtree: an r package for visualization and annotation of phylogenetic trees with their covariates and other associated data. Methods Ecol Evol. 2017;8:28-36. https://doi.org/1 $0.1111 / 2041-210 X .12628$

41. Andrews S. FastQC: a quality control tool for high throughput sequence data. 2010 Available online at: http://www.bioinformatics.babraham.ac.uk/ projects/fastqc.

42. Chen S, Zhou Y, Chen Y, Gu J. Fastp: An ultra-fast all-in-one FASTQ preprocessor. Bioinformatics. 2018:1884-90.

43. Kim D, Paggi JM, Park C, Bennett C, Salzberg SL. Graph-based genome alignment and genotyping with HISAT2 and HISAT-genotype. Nat Biotechnol. 2019;37:907-15. https://doi.org/10.1038/s41587-019-0201-4

44. Li H, Handsaker B, Wysoker A, Fennell T, Ruan J, Homer N, et al. The Sequence Alignment/Map format and SAMtools. Bioinformatics. 2009;25: 2078-9. https://doi.org/10.1093/bioinformatics/btp352.

45. Riga M, Denecke S, Livadaras I, Geibel S, Nauen R, Vontas J. Development of efficient RNAi in Nezara viridula for use in insecticide target discovery. Arch Insect Biochem Physiol. 2020;103:e21650. https://doi.org/10.1002/arch.21650.

46. Pearce SL, Clarke DF, East PD, Elfekih S, Gordon KHJ, Jermiin LS, et al. Genomic innovations, transcriptional plasticity and gene loss underlying the evolution and divergence of two highly polyphagous and invasive Helicoverpa pest species. BMC Biol. 2017;15:63. https://doi.org/10.1186/s1291 5-017-0402-6.

47. Denecke $\mathrm{S}$, loannidis $\mathrm{P}, \mathrm{Buer} \mathrm{B}$, Ilias $\mathrm{A}$, Douris $\mathrm{V}$, Topalis $\mathrm{P}$, et al. A transcriptomic and proteomic atlas of expression in the Nezara viridula (Heteroptera: Pentatomidae) midgut suggests the compartmentalization of xenobiotic metabolism and nutrient digestion. BMC Genomics. 2020;21:129. https://doi.org/10.1186/s12864-020-6459-6.

48. Rösner J, Merzendorfer H. Transcriptional plasticity of different ABC transporter genes from Tribolium castaneum contributes to diflubenzuron resistance. Insect Biochem Mol Biol. 2020;116:103282. https://doi.org/10.101 6/J.IBMB.2019.103282.

49. Kowalski $\mathrm{P}$, Baum $\mathrm{M}$, Körten $\mathrm{M}$, Donath $\mathrm{A}$, Dobler S. ABCB transporters in a leaf beetle respond to sequestered plant toxins. Proc R Soc B Biol Sci. 2020; 287:20201311. https://doi.org/10.1098/rspb.2020.1311

50. Mayer F, Mayer N, Chinn L, Pinsonneault RL, Kroetz D, Bainton RJ. Evolutionary conservation of vertebrate blood-brain barrier chemoprotective mechanisms in Drosophila. J Neurosci. 2009;29:3538-50. https://doi.org/10.1 523/JNEUROSCI.5564-08.2009.

51. Tian L, Yang J, Hou W, Xu B, Xie W, Wang S, et al. Molecular cloning and characterization of a P-glycoprotein from the diamondback moth, Plutella xylostella (Lepidoptera: Plutellidae). Int J Mol Sci. 2013;14:22891-905. https:// doi.org/10.3390/ijms141122891

52. Favell G, McNeil JN, Donly C. The ABCB Multidrug Resistance Proteins Do Not Contribute to Ivermectin Detoxification in the Colorado Potato Beetle, Leptinotarsa decemlineata (Say). Insects. 2020;11:135. https://doi.org/10.3390/ insects 11020135

53. Simmons J, D'Souza O, Rheault M, Donly C. Multidrug resistance protein gene expression in Trichoplusia ni caterpillars. Insect Mol Biol. 2013:62-71.

54. Jin M, Liao C, Chakrabarty S, Zheng W, Wu K, Xiao Y. Transcriptional response of ATP-binding cassette (ABC) transporters to insecticides in the cotton bollworm, Helicoverpa armigera. Pestic Biochem Physiol. 2018. https://doi.org/10.1016/J.PESTBP.2018.12.007.

55. Zuo Y, Huang J-L, Wang J, Feng Y, Han T-T, Wu Y-D, et al. Knockout of a Pglycoprotein gene increases susceptibility to abamectin and emamectin benzoate in Spodoptera exigua. Insect Mol Biol. 2017. https://doi.org/1 0.1111/imb.12338.

56. Aurade RM, Jayalakshmi SK, Sreeramulu K. P-glycoprotein ATPase from the resistant pest, Helicoverpa armigera: purification, characterization and effect of various insecticides on its transport function. Biochim Biophys Acta. 2010; 1798:1135-43. https://doi.org/10.1016/j.bbamem.2010.02.019.
57. Jin M, Cheng Y, Guo X, Li M, Chakrabarty S, Liu K, et al. Down-regulation of lysosomal protein ABCB6 increases gossypol susceptibility in Helicoverpa armigera. Insect Biochem Mol Biol. 2020;103387. https://doi.org/10.1016/j. ibmb.2020.103387.

58. Ohbayashi T, Takeshita K, Kitagawa W, Nikoh N, Koga R, Meng X-Y, et al. Insect's intestinal organ for symbiont sorting. Proc Natl Acad Sci U S A. 2015;112:E5179-88. https://doi.org/10.1073/pnas.1511454112.

\section{Publisher's Note}

Springer Nature remains neutral with regard to jurisdictional claims in published maps and institutional affiliations.
Ready to submit your research? Choose BMC and benefit from:

- fast, convenient online submission

- thorough peer review by experienced researchers in your field

- rapid publication on acceptance

- support for research data, including large and complex data types

- gold Open Access which fosters wider collaboration and increased citations

- maximum visibility for your research: over $100 \mathrm{M}$ website views per year

At BMC, research is always in progress.

Learn more biomedcentral.com/submissions 